\title{
Calculation of Complete Absorption and Intensity of Optical Radiation Spectrum of HeI $(\lambda=5875 \AA)$ with Fine Structure
}

\author{
Reda A. El-Koramy ${ }^{1}$, Nazer A. Ashurbecov² \\ ${ }^{1}$ Physics Department, Faculty of Science, Assiut University, Assiut, Egypt \\ ${ }^{2}$ Department Physical Electronics, Daghestan State University, Daghestan, Russia \\ E-mail:elkoramy@yahoo.com \\ Received February 13, 2011; revised April 24, 2011; accepted May 13, 2011
}

\begin{abstract}
Theoretical calculations which account for the complete absorption and intensity for the optical radiation $\mathrm{He}$ I $(\lambda=5875 \AA)$ spectral line with fine structure of the transition $2^{3} \mathrm{P}_{2,1,0}-3^{3} \mathrm{D}_{3,2,1}$ during He nanosecond discharge are presented. For different values of the absorption parameter $\left(\chi_{0} \ell\right)$, the absorption quantity $A$ of the three components distorted as a result of reabsorption multiple process has been numerically obtained and graphically presented. The theoretical results for small values of $\chi_{0} \ell(\leq 4)$ give a good agreement with the experimental data in literature.
\end{abstract}

Keywords: Optical Radiation Spectrum, Complete Absorption, Intracavity Laser Spectroscopy, Simpson's Formula, Computer Processing

\section{Introduction}

While the classical theories gave a semi-quantitative explanation for the interaction between matter and radiation, no precise and general treatment was possible before the advent of quantum theory. In principle, the quantum theory of matter allows one to calculate the various energy levels that any given atom or ion may possess. However, as long as classical electromagnetic theory is used, emitted, absorbed or scattered radiation is still ambiguous. Much detailed work remains to be done to elucidate the nature of numerous approximate treatments and to assess the ranges of applicability and the degree of approximation that has been achieved. The variation of inter-atomic (intermolecular) forces as a function of inter-atomic (inter-molecular) distance has been increasingly attracting the attention not only of theoretical and experimental physicists but also of those who are working on certain basic problems of chemistry, genetics and astrophysics.

Plasma-broadened and shifted spectral line profiles have been used for a number of years as a basis of an important non-interfering plasma diagnostic method. The numerous theoretical and experimental efforts have been made to find solid and reliable basis for this application. This technique became, in some cases, the most sensitive and often the only possible plasma diagnostic tool. In the early 1960s a number of attempts [1-3] were made to improve and to check experimentally existing theories of spectral line broadening by plasmas. Most of these early works were concerned with the Stark broadening of hydrogen lines. Owing to the large, linear Stark effect in hydrogen, these studies were very useful for plasma diagnostic purposes. However, it is not always convenient to seed plasma with hydrogen, and sometimes this is not possible.

Although the theory of Stark broadening was used only as a consistency check in the study of the influence of ion dynamics to the width and shift of visible helium lines in low electron density plasmas, an important conclusion was derived from the comparison with the results of semiclassical calculations $[4,5]$. This conclusion, which may be used for plasma diagnostic purposes with an average estimated accuracy of $\pm 20 \%$ and $\pm 30 \%$ for a neutral atom and singly charged ion lines, respectively, led to the fact that the total width and shift is a sum of the electron and all ion impact widths. On the basis of comparison of selected data in [4] and calculations for the energy positions and widths of singlet and triplet (even and odd) resonances of helium-like $(Z=2-10)$ systems lying between the $n=2$ and $n=3$ thresholds [6], an at- 
tempt was made to extend these works and to identify lines of various elements which may be used with higher accuracy for plasma diagnostic purposes [7].

The study of emission radiation from atoms (or ions) in plasma constitutes an important area of plasma diagnostics. From the experimental point of view, plasma is sometime optically thick to its emission lines, then, the emitted photons may be absorbed within the plasma. Over the past, more than thirty years period of formation and development of spectroscopic techniques, selective intra-cavity laser spectroscopy (ICLS) provides the possibility of simultaneously recording the profiles of a number of spectral lines and bands in probing both brightly emitting and nonradiative objects. Also, of particular interest, ICLS combines high sensitivity in detecting absorption centers [8-11] and, so, characterizes the integral absorption coefficient within the frequency limits of the absorption line [12,13]. Furthermore, transition probabilities and atomic lifetimes [4] and optical absorption and reflectance [14] have been reported. Predicted $\mathrm{He}$ I line intensities and line ratios in microwavegenerated plasmas and from a recently developed collisional-radiative model were compared with experiment $[15,16]$ It has been concluded that the intensity of triplet lines is strongly affected by the local metastable state $\left(2^{1} \mathrm{~S}\right.$ and $\left.2^{3} \mathrm{~S}\right)$ populations and the initial metstable fraction plays an important role in determining line intensities. In spite of there has been a lot of work done within the past decade, both theoretical and experimental, were given [17-23]; and, recently, resonance parameters and autoionization widths of the doubly excited states of the helium isoelectronic sequence [24] have been studied, however, there are some important aspects of the subject which have not been clearly discussed. Presently due to progresses of computer processing, the need of another review is quite obvious. The present work is merely to aid in this process of focusing thought upon crucial issues.

The present work is intended to derive analytic expressions for calculating dependencies of complete absorption and intensity of radiation spectrum on optical depth along the line sight in a nanosecond gas discharge. We report results of complete absorption for the optical radiation of $\mathrm{HeI}(\lambda=5875 \AA)$ spectral line with fine structure of the transition $2^{3} \mathrm{P}_{2,1,0}-3^{3} \mathrm{D}_{3,2,1}$ through $\mathrm{He}$ nanosecond discharge.

\section{Theoretical Analysis}

\subsection{Advanced Spectroscopic Treatment}

Spectroscopy is a powerful tool to diagnose plasmas and has played and will continue to play a major role in plasma physics research. Interest in line broadening has been renewed in the last few years because of the large amount of recent plasma work. The line shapes obtained by spectroscopic examination of the emission of plasma can yield information about plasma density.

\subsection{Doppler Producing Broadening and Shift of Spectral Lines}

The line profiles emitted from the plasma are governed by Doppler and Stark broadenings. Doppler effects give rise to further broadening rather than ones. So, other broadening mechanisms can be neglected for our plasma condition, which represent a typical emission spectrum from laboratory plasma. It consists of continuous bremsstrahlung and recombination radiation superposed by line radiation. We now visualize the plasma to be homogenous and to increase steadily its optical depth in the direction of observation. The spectral radiance will increase until it reaches the Planck value of the blackbody emission according to the existing temperature. We consider an emitting atom in motion to infinity and reduce temperature to the point where, classically at least, no translational motion exists. Every influence exerted on a radiating or absorbing atom (or molecule) modifies its spectral lines in one way or another. The effect of its own thermal motion, which broadens the lines statistically, is known as the Doppler Effect. In the case of emission or resonance absorption, the process of radiation damping, or a finite width of each of the two energy levels associated with spectral lines, is responsible for the natural width. If the atom is in a gas of the same kind, the spectral lines are broadened and sometimes are shifted with asymmetry of the line shape. The essential cause of line broadening lies in the finite difference of interaction energies of the radiating atom, in the initial and final states involved in the radiation process, with a colliding atom. When the interacting atoms, one of which is excited, are identical, a resonance case will be established. This resonance introduces twofold degeneracy (symmetric and anti-symmetric).

As it is known that the intensity of emission (absorption) is defined as the radiant energy emitted (absorbed), which penetrates a unit area in a plasma volume in unit time per unit solid angle perpendicular on the area, and, per unit of frequency $v$. The so-defined intensity encompasses radiation of all frequencies and hence, should be called "complete intensity". If one measures the spectral intensity of one line and its vicinity, the frequency (or wavelength) gives information that a certain element is present in a certain stage of ionization. The absorption and emission of a spectral line are determined by the population of the lower and the upper quantum level re- 
spectively, and, by Einstein's probabilities. For the calculation of the line intensities emitted from plasma, and, vice versa, for spectroscopic plasma diagnostics, the knowledge of the transition probabilities of all investigated lines is indispensable.

We suppose, our emitting atom has a velocity component $\xi$ in the line of sight of observer. Now, if the atom is emitting a spectral line radiation of wavelength $\lambda_{0}$, and a frequency $v_{0}$, Doppler effect causes our observer to receive radiation of wavelength $\lambda^{\prime}$, such that

$$
\lambda^{\prime}=\lambda_{0}\left(1-\frac{\xi}{c}\right),
$$

assuming $\xi \ll c$ (light velocity).

Velocity distribution in the line of sight is given by the Maxwellian distribution factor as $\exp \left[-\beta \xi^{2}\right]$, where

$$
\beta=M / 2 k T,
$$

[M-the atomic mass, $k$-Boltzmann's constant and $T$ is the absolute temperature].

From (1), it can be written

$$
\xi^{2}=\lambda_{0}^{2}(\Delta v)^{2}
$$

In the last relation $\lambda_{0}$ may now be considered as the wavelength corresponding to the maximum intensity of the line (line center), and $\Delta v$ as the frequency whose displacement corresponds to the line of the sight velocity $\xi$, or in other words, the line shift. Although there are some kinds of broadenings (such as Stark, van der Waals, ...etc), Doppler effects due to the relative motion of radiating systems give rise to further broadening rather than ones. If the velocities of radiating system have thermal distribution, collisions are negligible; otherwise, and furthermore, if one arbitrarily equates the line center intensity to unity, the Doppler effect alone produces a distribution of intensities over the spectral line which may be represented by the following form, using (3)

$$
I=I_{\circ} \exp \left[-\frac{M \lambda_{0}^{2}(\Delta v)^{2}}{2 k T}\right] .
$$

Doppler broadening results in a Gaussian line shape, whose half-width $\Delta v_{D}$ is defined as the frequency width of the spectral line at an intensity $I$ equivalent to one half the maximum intensity $\left(I_{0} / 2\right)$. Accordingly, using (4), we can write

$$
\Delta v_{D}=2\left(\frac{2 \ln 2 R T}{M}\right)^{1 / 2} \frac{v_{0}}{c},
$$

where $R$ represents the gas constant.

It may be remarked that the emitted spectrum of any homogenous source becomes more and more continuous

(smooth) as the depth of the source increases. This is due to the fact that the strongest lines tend to be reabsorbed, then the weaker ones and eventually also parts of the continuum, until the spectrum resembles a blackbody continuum.

\subsection{Spectroscopic Treatment of Radiation Absorption}

An observer collects radiation along the line of the sight and, in general, absorption of radiation will occur, and this has to be taken into account. This leads, neglecting scattering, to the following treatment. We assume an absorption line at the frequency $v$ with a dispersion profile and frequency-dependent absorption coefficient $\chi(v)$, such that its line width is much smaller than the generation line width. The generation spectrum corresponding to the entire pulse exhibits a valley which also has the dispersion profile described by the LambertBeers relation

$$
I(v)=\int_{0}^{\tau} I_{0}(v, t) \exp [-\chi(v) \ell] \mathrm{d} t
$$

Here: $I(v)$ represents the spectral radiance intensity of generation in the modes with narrow-band selective absorption (in the absence of saturation) (sometimes referred to as flow-out) recording from the absorbent 2 , as shown in the schematic diagram of Figure 1; $I_{0}(v, t)=$ $I_{0} f(t)$ - the spectral radiance intensity of generation in the absence of additional selective absorption, $f(t)$ is a function describing the shape of the radiation-pulse envelope in the spectral region (flow-in) at the absorbent surface 2 comes out of the radiant source 1 ; and $\ell=(c t x / L)$ is the effective thickness of the absorbing layer at the moment of time $t, x$ is the length of the absorbent, and $L$ is the cavity base. It must pointed out here that, in the particular case where a laser pulse can be approximated by a

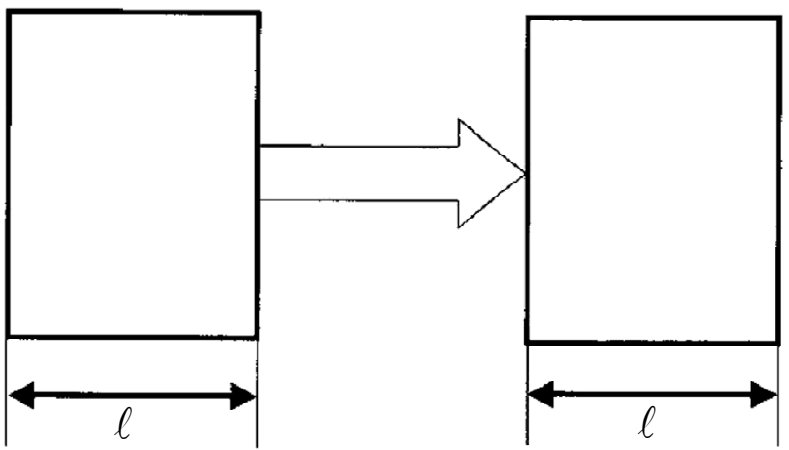

Figure 1. Schematic diagram of the illustrated positions of the 1-radiant source and 2-absorbent surface of a specimen. 
function which describes a rectangle, so,

$$
f(t)= \begin{cases}1 & 0 \leq t \leq \tau \\ 0 & t>\tau .\end{cases}
$$

Let us consider basic dependences and relationships which rather adequately describe intracavity absorption in pulsed lasers which are useful for measuring the magnitude of the frequency-dependent absorption introduced into the cavity. Under the conditions where the apparatus width of the spectral instrument used for recording the generation spectra of a laser with a selectively absorbing medium in the cavity is commensurable with the actual width of the absorption line or exceeds it, it is advisable to apply the method of complete absorption [13]. However, the basic aspects and special features of the measurements of the spectrum-integral absorption in the absorption line were given previously $[11,5]$.

The complete line absorption and intensity depend essentially on $\chi(v)$ and $\ell$.

The quantity of the complete absorption $A$ of radiation is defined as:

$$
A=1-\left[I(v) / I_{0}(v, t)\right] .
$$

\subsection{Limits of the Presented Treatment}

For simplicity, we restrict our attention to the following conditions concerning the spectrum generation:

1) We consider that the radiant source 1 has a rectangular form within its interval $\tau$, such that outside this interval no radiation may be emitted or absorbed, i.e.;

$$
I_{0}(v, t)=\left\{\begin{array}{ll}
I_{0}(v) & 0 \leq t \leq \tau \\
0 & t>\tau .
\end{array} .\right.
$$

2) On the other hand, since the absorption spectrum is different over the profile of the absorption line, the integration given in (6) extends over a limited frequency range of the absorbed line, such as

$$
I_{0}(v, t)= \begin{cases}I_{0}(v) & v_{1} \leq v \leq v_{2} \\ 0 & v>v_{2} .\end{cases}
$$

Here $\left(v_{2}-v_{1}\right)$ represents the frequency optical depth along the line of the sight, at which Doppler effect exhibits a constant value. Therefore, Equation (6) becomes:

$$
I(v)=\int_{v_{1}}^{v_{2}} I_{0}(v) \exp [-\chi(v) \ell] \mathrm{d} v
$$

If the radiated layer 1 and the absorbed one 2 (Figure 1) are identical in all its parameters [temperature, pressure, thickness and energy-level distribution function as well as the same identical atoms (or molecules) ...etc], so, for small values of $\chi_{0} \ell$ and using Equation (8), Eq- uation (7) takes the form [26]

$$
\begin{aligned}
& A=\int_{v_{1}}^{v_{2}} \frac{I_{0}(v)-I(v)}{I_{0}(v)} \mathrm{d} v, \text { or } \\
& A=\int_{v_{1}}^{v_{2}}\{1-\exp [-\chi(v) \ell]\} \mathrm{d} v,
\end{aligned}
$$

taking into consideration that $\chi(v)$ has the following expression [5]

$$
\chi(v)=\chi_{0} \exp \left[-2.7726\left(\frac{\left(v-v_{0}\right)}{\Delta v_{D}}\right)^{2}\right],
$$

where $\chi_{0}$ represents the absorption coefficient in the spectral line center and $\Delta v_{D}$ is, from its definition, taken at ordinate $\chi(v)=1 / 2 \chi_{0}$.

\section{Results and Discussion}

The choice of a transition involving only levels with well understood kinetics for this study is essential. The helium (He) discharge is one of the most thoroughly studied discharge systems [27]. We give now certain analysis needed for the calculation of the complete absorption of the fine structure HeI $(\lambda=5875 \AA)$ spectral line, obtained in a laboratory work [28] during a nanosecond $\mathrm{He}$ discharge, due to the optical transition $2^{3} \mathrm{P}_{2,1,0}-3^{3} \mathrm{D}_{3,2,1}$, schematic representation diagram of which is shown in Figure 2. This transition is ideal because of its convenient wavelength $(\lambda=5875 \AA)$, and most of the kinetics of the $3^{3} \mathrm{D}$ level [29], the $2^{3} \mathrm{P}$ level [30,31], and other nearby levels [32] are known. The energy levels ${ }^{3} \mathrm{D}_{3,2,1}$ of the state ${ }^{3} \mathrm{D}$ practically are coincident (splitting of that state consists of hundreds and thousands submultiplet fractions of percents) whereas the state $2{ }^{3} \mathrm{P}$ is reversal. By the rule of brightness, the intensities of $2{ }^{3} \mathrm{P}$ components
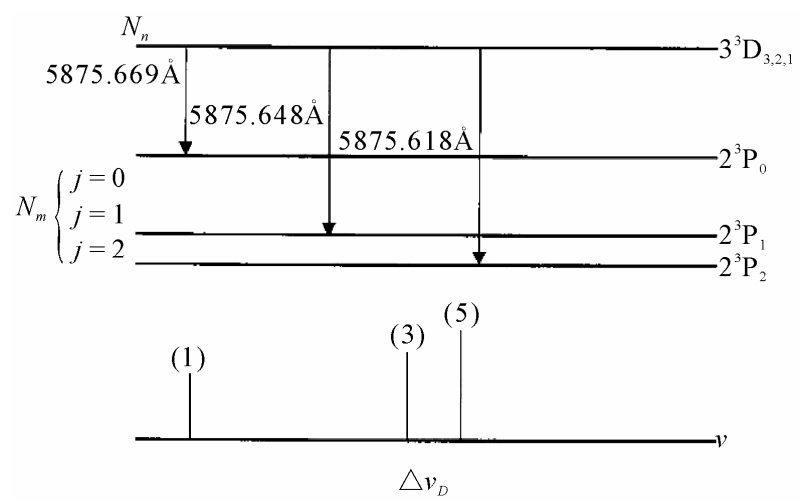

Figure 2. Schematic representation diagram of: a) the energy levels of the spectral line transition $2{ }^{3} \mathbf{P} 2,1,0-3$ ${ }^{3} \mathrm{D} 3,2,1$ of $\mathrm{HeI}(\lambda=5785 \AA)$, illustrating its fine structure and; b) the intensity ratios $(1: 3: 5)$ of its components with respect to their statistical weights. 
are in the ratios of 1:3:5, respectively, with their statistical weights of the levels $2^{3} \mathrm{P}_{0}, 2^{3} \mathrm{P}_{1}$ and $2^{3} \mathrm{P}_{2}$.

On the other hand, these ratios are the same as that observed between the absorption coefficients in the line center of each individual component. So, the relationship between $\chi_{0}$ and $I_{0}(v)$ of the stimulated emission at the frequency of the absorption band edge of the transition depends on the relative population of the energy levels $\left(N_{m} / N_{n}\right)$. Since the two energy levels $m$ and $n$ have a fine structure, for individual components $i \rightarrow j$, it may have the following relation [33]

$$
\chi(0)_{j i}=\frac{c^{2}}{2 h v_{i j}^{3}} \frac{g_{i}}{g_{j}} \frac{N_{j}}{N_{i}} I(0)_{i j},
$$

where $\chi(0)$ and $I(0)$ represent the absorption coefficient and intensity at the line center.

It is more easily established a resonance distribution for the nearest superposition sublevels rather than that for faraway remaining ones. Since, the frequencies of the three components, of which are close to each other, it can be used for the following substitutions:

$$
N_{j}=\frac{g_{j}}{g_{m}} N_{m}, N_{i}=\frac{g_{i}}{g_{n}} N_{n}
$$

and, therefore, Equation (11) may be re-written as

$$
\chi(0)_{j i}=\frac{c^{2}}{2 h v_{i j}^{3}} \frac{g_{n}}{g_{m}} \frac{N_{m}}{N_{n}} I(0)_{i j}
$$

If $\frac{N_{m}}{N_{n}}$ is constant, the absorption coefficients of the spectral line components are proportional to its brightness. This partial fulfillment of Boltzmann's law also allows one to keep the processing results of measurements to be carried out for lines with a fine structure since $N_{m} / N_{n}$ is constant during the experimental conditions. Neglecting splitting of the upper-level, so, for the summation of the transition probability $A_{n m}$ and the transition probability of the individual components, it may be written in the following form

$$
A_{n j}=A_{n m} \frac{a_{j}}{\sum_{j} a_{j}},
$$

where $A_{n 1}: A_{n 2}: A_{n 3}=a_{1}: a_{2}: a_{3}=1: 3: 5$; and, according to the rule of brightness, then $a_{j}=g_{j}$, where $g_{j}$ represents the statistical weights of the sub-levels on which the level -m splits.

In the experimental conditions, one mirror has been used for the radiant source 1 . So, if we suppose that $I^{\prime}$ represents the intensity of the flow-in radiation, then, taking into account the re-absorption and loss on the front window, $r I^{\prime}$ represents the reflected flow-in inten- sity, where $r$ is called the reflection coefficient of the mirror. We suppose that the defined relationships of initial intensities of the three components and their absorption coefficients in the line center, respectively, are as follows

$$
\begin{aligned}
& I_{01}: I_{02}: I_{03}=a_{1}: a_{2}: a_{3} ; \\
& \chi_{01}: \chi_{02}: \chi_{03}=b_{1}: b_{2}: b_{3} ;
\end{aligned}
$$

i.e., generally speaking,

$$
I_{0 j}=C a_{j}, \chi_{0 j}=C^{\prime} b_{j}
$$

where $C$ and $C^{\prime}$ are constants in the experimental conditions, therefore, the intensity distribution function of the $\mathrm{j}$-th component takes the following form [33]

$$
\begin{aligned}
& I_{j}(v) \\
& =r \frac{C}{C^{\prime}} \frac{a_{0 j}}{b_{0 j}}\left(1-\exp \left\{-C^{\prime} b_{0 j} \ell \exp \left[-\beta c^{2}\left(\frac{v-v_{0 j}}{v_{0 j}}\right)^{2}\right]\right\}\right)
\end{aligned}
$$

Accordingly, the intensity distribution function of the sum flow-in radiation from all components can be written as

$$
\begin{aligned}
& I^{\prime}(v) \\
& =r \frac{C}{C^{\prime}} \sum_{j} \frac{a_{0 j}}{b_{0 j}}\left(1-\exp \left\{-C^{\prime} b_{0 j} \ell \exp \left[-\beta c^{2}\left(\frac{v-v_{0 j}}{v_{0 j}}\right)^{2}\right]\right\}\right)
\end{aligned}
$$

On the other hand, during the passing of the absorbent source 2, a part of the radiant flow-in would be absorbed, so, $\chi(v)$, defined in Equation (10), must be equated with the sum of the absorption coefficients of all individual components, i.e.,

$$
\chi(v)=\sum_{j} \chi_{j}(v),
$$

and, for the radiant flow-out,

$$
I^{\prime \prime}(v)=I^{\prime}(v) \exp \left[-\sum_{j} \chi_{j}(v) \ell\right] .
$$

As it is known, an integrated intensity equals the area $S(v)$ under the curve of the corresponding intensity distribution function. Figure 3 shows, experimentally, the re-absorption contours of the two components of investigated HeI $(\lambda=5875 \AA)$. Then, assuming that $I^{\prime}(v)$ corresponding to the sum intensities for the three components distorted as a result of re-absorption during one multiple passage is proportional to its corresponding area $S^{\prime}(v)$, and also $S^{\prime \prime}(v)$ corresponding to $I^{\prime \prime}(v)$, the definition of the complete absorption coefficient [Equation (9)], may be given as 


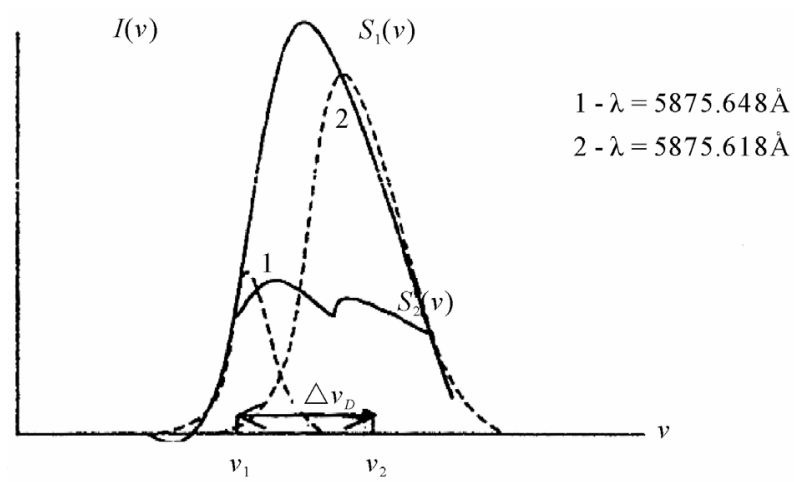

[ $S_{1}(v)$ and $S_{2}(v)$ represent sum contours of curves $1 \& 2$; after first and second absorption processes, respectively]

Figure 3. The absorption and re-absorption contours of two components of fine structure of the investigated HeI $(\lambda=$ $5875 \AA$ A).

$$
A=\frac{S^{\prime}(v)-S^{\prime \prime}(v)}{S^{\prime}(v)}=\frac{I^{\prime}(v)-I^{\prime \prime}(v)}{I^{\prime}(v)} .
$$

It should be pointed-out here that we have fitted a superposition of three Voigt profiles to the measured line profiles. The frequency and relative intensities of the three fine-structure components are used for the Voigt profiles. Each of the Voigt profiles consists of the appropriate Doppler and apparatus profile.

Substituting Equations (15) and (16) for Equation (17), and taking into account Equation (9), we finally, have

$$
A=1-\frac{\int_{v_{1}}^{v_{2}} \sum_{j}\left[\mathrm{e}^{-\sum_{j} \chi_{j}(v) \ell}-\mathrm{e}^{-\left\{\chi_{j}(v) \ell+\sum_{j} \chi_{j}(v) \ell\right\}}\right] \mathrm{d} v}{\int_{v_{1}}^{v_{2}} \sum_{j}\left(1-\mathrm{e}^{-\chi_{j}(v) \ell}\right) \mathrm{d} v} .
$$

The numerical calculations of the dependencies of $A$, using Equation (18), on $\chi_{0} \ell$ have been made for the investigated HeI spectral line with or without its fine structure, with the aid of Simpson's formula [34] and computer processing. In the calculated program, the following relations and values have been considered:

$$
\begin{aligned}
& \text { 1) } \chi_{j}(v) \ell=\chi_{0 j} \ell \mathrm{e}^{-2.7726\left(\frac{v-v_{j}}{\Delta v_{D j}}\right)^{2}}, j=1,2,3 ; \\
& \chi_{01} \ell=\frac{1}{9} \chi_{0} \ell ; \chi_{02} \ell=\frac{3}{9} \chi_{0} \ell ; \chi_{03} \ell=\frac{5}{9} \chi_{0} \ell
\end{aligned}
$$

2) The wavelengths of the components of the fine structure and its corresponding frequencies and Doppler half-widths calculated by using Equation (5) are as follows

$$
\lambda_{1}=5875.669 \AA, v_{1}=5.1057995 \cdot 10^{14} \mathrm{~s}^{-1}, \Delta v_{D 1}=
$$
$3.1659745 \cdot 10^{9} \mathrm{~s}^{-1}$;

$\lambda_{2}=5875.648 \AA, v_{2}=5.1058138 \cdot 10^{14} \mathrm{~s}^{-1}, \Delta v_{D 2}=$ $3.1659844 \cdot 10^{9} \mathrm{~s}^{-1}$; $\lambda_{3}=5875.618 \AA, v_{3}=5.1058458 \cdot 10^{14} \mathrm{~s}^{-1}, \Delta v_{D 3}=$ $3.1660042 \cdot 10^{9} \mathrm{~s}^{-1}$;

3) For the natural spectral line, we have

$\lambda_{0}=5875.000 \AA, v_{0}=5.1063809 \cdot 10^{14} \mathrm{~s}^{-1}, \Delta v_{D 0}=$ $3.1663351 \cdot 10^{9} \mathrm{~s}^{-1}$.

The obtained results of the relation between $A$ and $\chi_{0} \ell$ are represented in Figure 4. From the graphs of that figure, one can see that, at small values of $\chi_{0} \ell$, there is a great loss $(\sim$ twice $)$ in the absorption process for the investigated spectral line with its fine structure. Furthermore, with the increasing layer thickness, the fine structure behaves at much smaller values in $A$ rather than that in the natural spectral line. This result should be taken into account, since the population is spread from one line to three with close but well resolved frequencies.

The results of comparison with semiclassical calculations for neutral atom lines [4] and Monte Carlo simulation for radiation re-absorption [35] are very encouraging. For a number of $\mathrm{He}$ I, C I, N I, O I and F I lines the agreement is well within $\pm 20 \%$ and in several cases, $\mathrm{He}$ I and C I, better than $\pm 15 \%$. In comparison with neutrals, for singly ionized atom lines the results of the test experiment versus semiclassical calculations [4] are rather meager. The results for multiply ionized atom lines are a pleasant surprise. The average deviation between semiclassical electron impact calculations [36] does not exceed $20 \%$ and in several cases is smaller than $10 \%$. If one takes into account the unsettled situation for $\mathrm{Ca}$ II, Si II and Xe II, it seems that a lot of theoretical and experimental effort has to be involved to improve the present situation.

\section{Conclusions}

The main conclusion to be drawn from these results is that the absorption of fine structure of the investigated spectral line occurs in a certain frequency range around $v_{0}$ owing to various processes in the thermal motion of

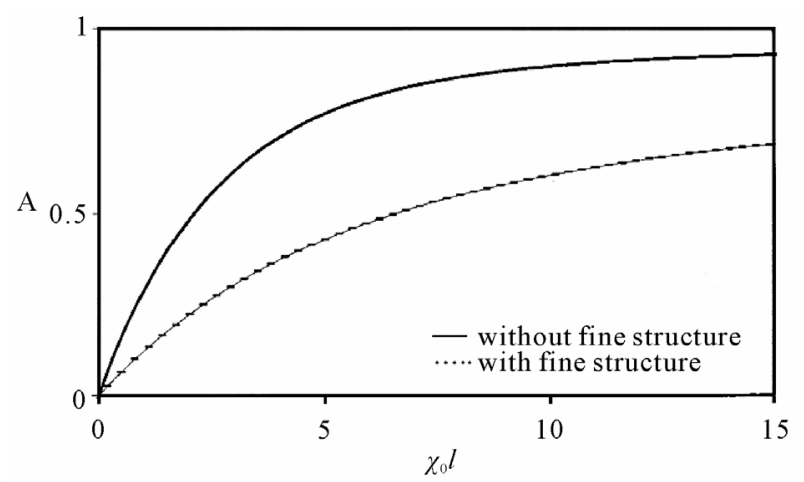

Figure 4. Dependence of the calculated complete absorption $A$ of the investigated HeI $(\lambda=5875 \AA)$ on the optical parameter $\chi_{0} \ell$, with and without fine structure. 
the gas atoms. Apart from its intrinsic interest in the study of Doppler broadening, the phenomenon described essentially in this review is important in connection with the limitation of the resolution close spectral lines. This limitation arises in a two-fold manner; the absorption is reduced, making the lines more difficult to observe, but this reduction is much less at the edge of a line than in the center.

Another specific conclusion is that multiplets are very useful in the assessment of the optical depth. The intensity ratio of their components usually is well known for the free atoms and ions, and any deviation will indicate the magnitude of the self-absorption, the strongest component being affected. Within limits the optical depths of the individual components can even be derived.

The results of the present work predict that, if plasma is seeded with atomic species having high absorption coefficients for ground states atoms or ions, the respective resonance transitions easily become optically thick and their spectral radiance allows a temperature measurement. With respect to applications, these plasmas can be used thus as high-power sources radiating at the blackbody radiance in narrow spectral regions [37].

Furthermore, the results of this work support the assumption made in its theoretical analysis that the line broadening due to Doppler effects varies directly the absorbed layer thickness. So, it is more convenient in an experimental work of plasma laboratory to make the absorbent layer thickness as small as possible to avoid the greater loss in the emitted radiation. On the other hand, it is pointed out here that Doppler broadening calculations have made possible a different and very convenient approach for determining the electron densities in dense plasmas with an accuracy comparable to other spectroscopic methods [38].

Finally, the theoretical results of the calculations offer a satisfactory agreement with the experimental data in literature [20]. To extend the study to other ions of the sequence, plasmas with known density and temperature especially at higher values and/or spectrometers with higher resolution are necessary.

\section{Acknowledgements}

The authors wish to express his gratitude to Prof. Dr. Salama A. A, Professor of Numerical Analysis at Assiut University, Assiut, Egypt for his guidance and valuable assistance in the statistical calculations.

\section{References}

[1] H. R. Griem, M. Baranger, A. C. Kolb and G. Oertel, "Stark Broadening of Neutral Helium Lines in a Plasma," Physical Review, Vol. 125, No. 1, 1962, pp. 177-195.

\section{doi:10.1103/PhysRev.125.177}

[2] H. R. Griem, "Stark Broadening of Isolated Spectral Lines from Heavy Elements in a Plasma," Physical Review, Vol. 128, No. 2, 1965, pp. 515-523. doi:10.1103/PhysRev.128.515

[3] W. Lochte-Holtgreven, "Plasma Diagnistics," NorthHolland Company, Amsterdam, 1968.

[4] H. R. Griem, "Spectral Line Broadening by Plasmas," Academic Press, Inc., New York, 1974.

[5] A. N. Rubin and M. V. Belokon, "Influence of the Concentration of the Absorbent on the Depth of a Dip in Stimulated Emission Spectrum of a Dye Laser Used in Measurement of Weak Absorption by the Intracavity Spectroscopy Method," Soviet Journal of Quantum Electronics, Vol. 6, No. 1, 1976, pp. 79-81. doi:10.1070/QE1976v006n01ABEH010823

[6] H. Bachau, F. Martin, A. Martin, A. Riera and M. Yanez, "Resonance Parameters and Properties of Helium-Like Doubly Excited States $2 \leq \mathrm{z} \leq 10$," Atomic Data and $\mathrm{Nu}$ clear Data Tables, Vol. 48, No. 2, 1991, pp. 167-212. doi:10.1016/0092-640X(91)90006-P

[7] N. Konjevic, "Plasma Broadening and Shifting of Non-Hydrogenic Spectral Lines: Present States and Applications," Physics Reports, Vol. 316, No. 6, 1999, pp. 339-401. doi:10.1016/S0370-1573(98)00132-X

[8] G. Torsi, E. Desimoni, F. Palmisano and L. Sabbatini, "Determination of Lead in Air by Electro-Thermal Atomic Spectroscopy with Electrostatic Accumulation Furnance", Analytical Chemistry, Vol. 53, No. 7, 1981, pp. 1035-1038. doi:10.1021/ac00230a026

[9] S. G. Harris, "Intracavity Laser Spectroscopy: An Old Field with New Prospects for Combustion Diagnostics," Applied Optics, Vol. 23, No. 9, 1984, pp. 1311-1318. doi:10.1364/AO.23.001311

[10] A. P. Voitovich, A. Ch. Voitovich and V. V. Mashko, "Resonance Polarization Phenomena in Broadband Lasers with Anisotropic Absorbing Gas Media," Laser Physics, Vol. 5, No. 5, 1995, pp. 927-951.

[11] V. M. Baev, T. Latz and P. E. Toschek, "Laser Intracavity Absorption Spectroscopy," Applied Physics B, Vol. 69, No. 3, 1999, pp. 171-202. doi:10.1007/s003400050793

[12] J. Workman Jr. and A. W. Springsteen, "Applied Spectroscopy," Academic Press, New York, 1998.

[13] V. S. Burakov and S. N. J. Raikov, "Intracavity Laser Spectroscopy: Plasma Diagnostics and Spectral Analysis," Journal of Applied Spectroscopy, Vol. 69, No. 4, 2002, pp. 492-518. doi:10.1023/A:1020639728912

[14] Y. Toyozawa, "Optical Absorption and Reflectance," Encyclopedia of Condensed. Matter Physics, 2005, pp. 142-147.

[15] K. Shin, O. Noriyastu, T. Shuichi and N. Tomohide, "Comparison of He I Line Intensit Ratio Method and Electrostatic Probe for Electron Density and Temperature Measurements in NAGDIS-II," Physics of Plasmas, Vol. 13, No. 1, 2006, p. 13301.

[16] J.-W. Ahn, D. Craig, G. Flksel, D. J. Den Hartag and J. K. 
Anderson, "Emission Intensities and Line Ratios from a Fast Neutral Helium Beam," Physics of Plasmas, Vol. 14, No. 8, 2007, p. 3301 (10 pages).

[17] J. L. McHale, "Molecular Spectroscopy," Prentice Hall, Englewood Cliffs, 1998.

[18] Y. Toshimitsu, M. Norio, S. H. Ryugo, W. Eberhard and E. John, "Antiprotonic Helium," Physics Reports, Vol. 366, No. 4-5, 2002, pp. 183-187. doi:10.1016/S0370-1573(01)00082-5

[19] J. M. Hollas, "Modern Spectroscopy," 4th Edition, Jon Wiley \& Sons, New York, 2004.

[20] V. V. Grishachev, V. I. Denisov, V. G. Zhotikov, V. N. Kuryatov and E. F. Nasedkin, "New Potentialities of Intracavity Spectroscopy of Matter Using Counterpropagating Wave in a Ring Laser," Opitics and Spectroscopy: Physical and Quantum Optics, Vol. 98, No. 1, 2005, pp. 47-52.

[21] V. I. Denisov, V. V. Grishachev, V. N. Kuryatov, E. F. Nasedkin and V. G. Zhotikov, "The Ultra-High Resolution Sensitivity by Spectral Measurement on the Basis of Laser," Journal of Quantitative Spectroscopy \& Radiation Transfers, Vol. 103, No. 2, 2007, pp. 302-313. doi:10.1016/j.jqsrt.2006.02.057

[22] C. S. Matthew, J. T. Michael, A. Pe'er, J. Ye, E. S. Jason, G. Vladislav and A. D. Scott, "Direct Frequency Comb Spectroscopy," Advances in Atomic, Molecular, and Optical Physics, Vol. 55, 2008, pp. 1-60. doi:10.1016/S1049-250X(07)55001-9

[23] S. Djenize, "Stark Broadening in the Sb III Spectrum," Physics Letters A, Vol. 372, No. 44, 2008, pp. 66586660.

[24] S. I. Themelis, "Resonance Energies and Auto-Ionization Widths $2 \mathrm{p}^{2}{ }^{1} \mathrm{D}^{\mathrm{e}}, 3 \mathrm{~d}^{2}{ }^{1} \mathrm{G}^{\mathrm{e}}$ and $4 \mathrm{f}^{2}{ }^{1} \mathrm{I}^{\mathrm{e}}$ Doubly Excited States of the Helium Iso-electric Sequence," Physics Letters A, Vol. 374, No. 44, 2010, pp. 4512-4516. doi:10.1016/j.physleta.2010.09.021

[25] E. A. Sviridenkovinitsa (Ed.) "Intracavity Laser Spectroscopy," Proceedings of SPIE, 1998.

[26] R. A. El-Koramy, "Transversal Magnetic Field Effect on the Populations of Excited States of Helium Atoms in Nanosecond Discharge," International Journal of Modern Physics B, Vol. 18, No. 3, 2004, pp. 395-408. doi:10.1142/S0217979204018503

[27] R. Deloche, P. Monchicourt, M. Cheret and F. Lambert, "High-Pressure Helium after Glow at Room Temperature," Physical Review A, Vol. 13, No. 3, 1976, pp. 11401176. doi:10.1103/PhysRevA.13.1140
[28] N. A. Ashurbecov, V. S. Kurbanismailov, O. A. Omarov and N. O. Omarova, "The Kinetics of Excited Atoms and Optical Radiation under Conditions of Wave Mechanism of Breakdown in Inert Gases," High Temperature, Vol. 38, No. 5, 2000, pp. 795-810. doi:10.1007/BF02755936

[29] J.-C. Gauthler, F. Devos and J. F. Delpech, "Temperature Dependence of the Collisional Relaxation Processes for the $\mathrm{n}=3$ Triplet States of Helium," Physical Review A, Vol. 14, No. 6, 1976, pp. 2182-2189. doi:10.1103/PhysRevA.14.2182

[30] J. E. Lawler, J. W. Parker, L. W. Anderson and W. A. Fitzsimmons, "Helium $3{ }^{3} \mathrm{~S}$ Decay Rates in a High-Pressure after Glow," Physical Review A, Vol. 19, No. 1 1979, pp. 156-159.

[31] J. E. Lawler, J. W. Parker, L. W. Anderson and W. A. Fitzsimmons, "Nanosecond Time-Resolved Spectroscopy of the $n=2$ Levels in a High-Pressure He Discharge," Physical Review Letters, Vol. 39, No. 9, 1977, pp. 543546. doi:10.1103/PhysRevLett.39.543

[32] S. Kubota, C. Davies and T. A. King, "Relaxation of He $\left(3{ }^{1} \mathrm{~S}, 3^{3} \mathrm{~S}, 3^{3} \mathrm{P}\right)$ States in $\mathrm{Ne}, \mathrm{Ar}, \mathrm{Kr}$ and Xe Collisions," Journal of Physics B: Atomic, Molecular and Optical Physics, Vol. 8, No. 8, 1975, pp. 1220-1227.

[33] S. E. Frish, "Spektroskopiya Gazorazeryadnoi Plasmy," (Spectroscopy of Gas-Discharge Plasma), Nauka, Moscow, 1970.

[34] N. C. Bakhvalov, "Numerical Methods," Nauka, Moscow, 1973

[35] M. Seo, M. Nimura, M. Hasuo and T. Fujimoto, "Disalignment of Excited Atoms by Radiation Reabsorption: Neon $2 \mathrm{p}_{2}$ Atoms in a Discharge Plasma," Journal of Physics B, Vol. 36, No. 9, 2003, pp. 1869-1884. doi:10.1088/0953-4075/36/9/316

[36] M. S. Dimitrijevi, "A Program to Provide Stark-Broadening Data for Stellar and Laboratory Plasma," Journal of Applied Spectroscopy, Vol. 63, No. 5, 1996, pp. 684-689. doi:10.1007/BF02606861

[37] F. Bottcher, U. Ackermann and H.-J. Kunze, "Gas-Linear Pinch as a Source of Blackbody-Limited VUV Line Radiation", Applied Optics, Vol. 25, No. 18, 1986, pp. 3307-3311._doi:10.1364/AO.25.003307

[38] K. H. Finken, G. Bertschinge, S. Maurmann., H.-J. Kunze, "Spectroscopic Investigation of High-Density Plasmas Produced in a z-Pinch," Journal of Quantitative Spectroscopy and Radiation Transfers, Vol. 20, No, 5, 1978, pp. 467-476. doi:10.1016/0022-4073(78)90050-X 